\title{
A Machine Learning Approach for Temporal Vibration Analysis Applied to Predictive Maintenance
}

\author{
Roberto Alexandre Dias \\ Federal Institute of Santa \\ Catarina - IFSC \\ 950 Av. Mauro Ramos \\ Florianópolis, SC, Brazil
}

\author{
Pedro Von Hertwig \\ Federal Institute of Santa \\ Catarina - IFSC \\ 950 Av. Mauro Ramos \\ Florianópolis, SC, Brazil
}

\author{
Mário de Noronha Neto \\ Federal Institute of Santa Catarina \\ - IFSC \\ 950 Av. Mauro Ramos \\ Florianópolis, SC, Brazil
}

\begin{abstract}
The operational condition of a machine affects the quality and efficiency of its work, and letting a problem arrive in a critical state results in negative consequences, which can cause equipment loss and extensive downtime in a factory. The maintenance of machines, therefore, is a concern that came to exist along with the creation of the industry. This work shows the development of a platform that takes advantage of recent advances in sensor technology and machine learning to assist the predictive maintenance process, identifying problems in advance before serious failures can occur. The work proposes a supervisory system which receives high frequency vibration data, stores it, and analyzes the functioning of a machine to classify its behavior as normal or anomalous, generating alerts. The results achieved show that it is appropriate to use machine learning to monitor machines, since well-structured algorithms can detect possible problems before they become apparent to humans.
\end{abstract}

\section{General Terms}

Predictive maintenance of electrical machines. Vibration monitoring. Machine learning

\section{Keywords}

Vibration analyses. Temporal analysis. Predictive maintenance Machine learning.

\section{INTRODUCTION}

A deterioration of equipment conditions leads to deviations in the production process and a drop in quality. Only proper maintenance can guarantee that the process will not lose quality due to deviations caused by the equipment [1].

In addition to the drop in product quality, the reduction in productivity due to unscheduled downtime is an obvious harm of the lack of proper maintenance. Less obvious is the drop in productivity even if the equipment is not stopped. This condition leads the company to look for the origin of the deficiency in factors such as tooling, materials, and operators; increasing the operational costs in a measurable way, and having effects of non-measurable costs, such as the erosion of the company's image. [1].

Machines are subject to failure in their operation, which incurs high costs due to the replacement of more parts and time lost while a repair is carried out.

Preventive maintenance, which takes place at fixed intervals of time according to a manufacturer's recommendation, is one of the maintenance options. It involves changing parts before the time of failure, to reduce the failure rate of the equipment. This practice, however, is not the most efficient because the intervention in healthy equipment generates costs that can be avoided.
Predictive maintenance, in contrast, is based on the principle of analyzing the machine's operating conditions to determine whether intervention is required. Thus, maintenance costs are restricted to equipment that in fact presents the possibility of imminent failure.

Vibration analysis for diagnosis and condition evaluation has a long history of application to mechanical and energy equipment. Many types of defects increase the vibration level of machines or change their behavior in some way [2].

In this context, "subjective monitoring" is the monitoring that is performed by maintenance personnel using their senses when a mechanic touches a housing, for example, he can perceive its vibration. Thus, a more experienced mechanic can provide greater precision in diagnosing a machine [3]. Such monitoring techniques, however, have numerous shortcomings: the diagnosis is subjective, depends on experienced personnel and does not provide continuous analysis.

In recent years, electrical machine condition monitoring systems have become increasingly efficient and sophisticated. For autonomous monitoring, the vibration must be converted into an electrical signal. Some types of hardware that can be used are piezoelectric accelerometers and MEMS (Micro Electromechanical Systems) [4]. The increasing automation of detection processes has made automated diagnostic and prognostic systems a valuable tool for maintenance personnel and can even replace humans [5]. In this context, machine learning is a tool to aid in the decision-making process that has been gaining popularity due to its ease of adaptation to unfamiliar scenarios and the ability to solve difficult problems solved through simple mathematical modeling. Machine learning approaches have already been used successfully to identify failures in rotating machines [6].

The present work aims to explore the field of using machine learning for application in predictive maintenance.

\section{BIBLIOGRAPHIC REVIEW}

\subsection{Failure prediction methods}

According to [5] existing methods of failure prediction can be grouped into three main categories:

- Traditional reliability method (prediction based on past events - preventive maintenance)

- Prognostic method (prediction based on condition monitoring)

- Integrated method (prediction based on past events and condition monitoring) 
The first and last category, which involve the statistical distribution of past events, are not always possible because data from past events are only useful for other equipment in identical working conditions [5]. Machines in the industry often operate under unique conditions and do not have such historical data available.

For the prognostic method based on condition monitoring, the predictions are further divided into three subcategories [7]:

- Physical model

- $\quad$ Data-driven model

- Hybrid models, which combine the two previous categories.

The prediction based on condition with a physical model (and, by extension, also with a hybrid model) implies the construction of a comprehensive mathematical model that describes the physical relationships of the system and its failure modes. This brings some advantages to the process; notably, as physical models do not depend on comparing training data, errors associated with data extrapolation are reduced and the model is immediately effective. There is also the possibility to simulate scenarios for which data collection would be rare or difficult, and to determine the operation of the machine under adverse conditions [8].

However, these models have little scalability and the adoption of physical modeling is expensive due to the fact that each monitored machine requires a detailed model of its normal operation and failure modes. The model is affected in a complex way by the operating condition to which the machine in question is subjected and by the interaction between its internal components.

In contrast to this, a data-driven model has less need for specific knowledge of the domain in which the machine is inserted, has greater simplicity, scalability, and reduced development cost. Another advantage is the fact that once trained, its use is computationally more efficient than that of physical models [9].

\subsection{Indicative factors of machine health}

From the raw vibration data, it is possible to process the signal to obtain some characteristics that tell us more than just the intensity of vibration at a given time. Some of them are:

- RMS - root mean square, is a characteristic that measures the energy in a signature vibration. It can be highly effective in detecting unbalance in rotating systems [2].

- Kurtosis - is defined as the fourth moment of distribution and measures how smooth or pointed the distribution is, in relation to a normal distribution. It is indicative of large peaks in the data set [2].

- Peak to peak - difference between highest and lowest recorded values. It gives an idea of the amplitude of the vibration signal.

- Crest factor - defined as the largest peak in the window divided by the RMS value, a high crest factor indicates amplitude values well above the average present in the data set.

These characteristics are scalar obtained through the application of a mathematical operation over several points in a time series. Thus, they can be more directly compared between windows of the same size at different times.

\section{PROBLEM DEFINITION}

Rotating electrical machines are widely used in industries such as processing, oil, and gas. These industries need machines to operate continuously at an optimum level. The performance of such machines is mainly dependent on the condition of their components such as bearings, transmissions, pumps, compressors, engines, and generators [6].

In the industry, the application of preventive maintenance occurs through experience or recommendations from the equipment supplier and is based on a scientific statistical approach. In most cases, it is performed at regular time intervals [10]. However, preventive maintenance plans copied from the manufacturer's manual in general are not applicable because [11]:

- $\quad$ Each machine works under different conditions and environments, and therefore requires different plans.

- Machine designers often do not have the same experience with machine failures and preventive modes as those who operate and maintain them.

- Machine suppliers may have a hidden agenda for maximizing parts exchange through plans that recommend more frequent maintenance than necessary.

Predictive maintenance, unlike preventive maintenance, is characterized by the measurement and analysis of machine variables that can predict a possible failure. With this, the maintenance team can plan for the intervention and purchase of parts (maintenance cost), reducing inventory expenses and avoiding unnecessary stops [1].

For condition-based predictive maintenance, there are three key elements: (i) the collection and storage of information, (ii) the conditioning and extraction of learning attributes for acquired data, and (iii) the decision process for recommending actions maintenance through fault diagnosis or prognosis. [5].

The present work aims to develop an algorithm regarding the last two points above, adopting the point of view of a system that receives vibration data from any source, preferably in real time. From this data, the algorithm must predict the imminence of a failure.

\section{PROPOSED SOLUTION}

In view of the advantages of predictive maintenance based on vibration analysis and prioritizing the flexibility of the algorithm to be applicable to different situations in the industry, the present work will implement an algorithm and platform aimed at vibration analysis for maintenance based on condition oriented to data.

A platform must receive continuous data from a given electrical machine to train its condition monitoring model.

For the development and reliability test of the developed algorithms, this work will be used as reference the data set referring to a test bench provided by NASA [12]. In the test, bearings were subjected to a radial load until they reached a point of failure. An example of the raw data contained in the experiment can be seen in Figure 1 


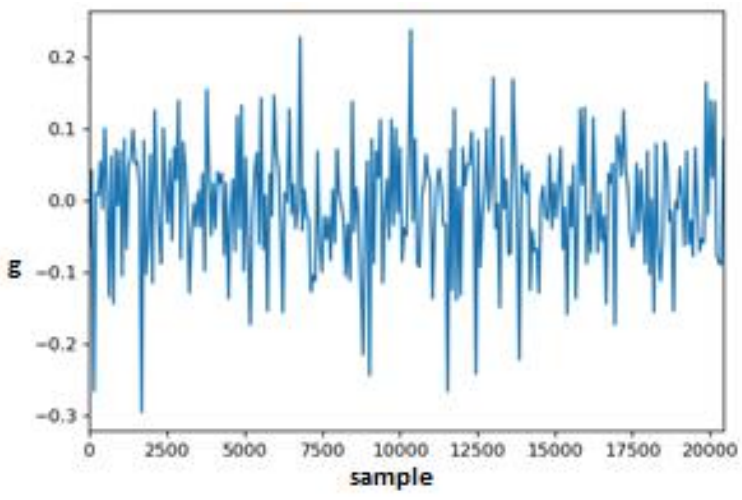

Figure 1 - Visualization for one second of raw data.

The graph shows the acceleration data recorded by one of the sensors, for an interval of 1 second. The $\mathrm{X}$ axis indicates the measurement number, made at an acquisition rate of $20 \mathrm{kHz}$. The data set consists of windows like this, 1 second each, recorded at 10-minute intervals.

\subsection{Time classifiers and aggregate characteristics}

One approach of detecting points outside the curve is the temporal detection of anomalies, which is concerned with data over time. In this context, there are still several subdivisions of methodologies that can be used: time or multidimensional series, point-to-point, or windowed analysis, continuous or discrete series, and availability or non-availability of previous anomalous data. [13]. Thus, the presented solution will explore the methodologies that use the series of points in chronological order (time series) that comes from the vibration signals of a monitored electric machine.

In Figure 2 we can see the result of processing the characteristics applied to the data set provided by NASA. In this case, windows of one second with an acquisition frequency of $20 \mathrm{kHz}$ were computed and measurements made every 10 minutes.

The caption "Ch 1 ... 4" refers to the measurement channels (accelerometers for each of the 4 monitored bearings).
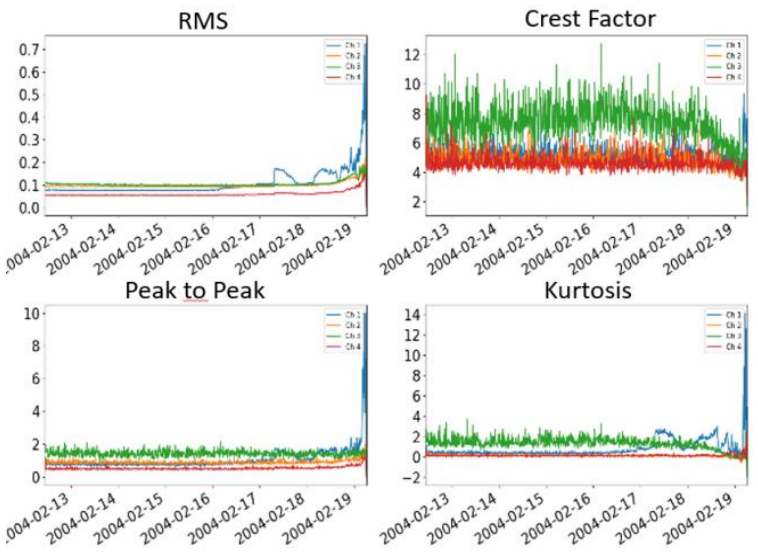

Figure 2 - Scalar under windows

\subsection{Grouping classifiers}

The solution developed, therefore, can benefit from different approaches, each with advantages and disadvantages. Fortunately, work developed in the field of machine learning is not necessarily restricted to one method or another; in the case of the present work, the temporal analysis or analysis of scalar characteristics aggregated over time.

Clusters are sets of classifiers that aim to increase the reliability of a system through the union of several models. A grouper, also called a multiple classifier or committee, is a set of individual classifiers whose predictions are combined to create a new prediction. Clusters have been shown to be an efficient way to improve the accuracy of a complex problem, transforming it into several subproblems. [14].

Figure 3 shows the hierarchy of elements present in a possible grouping system for vibration-based maintenance.

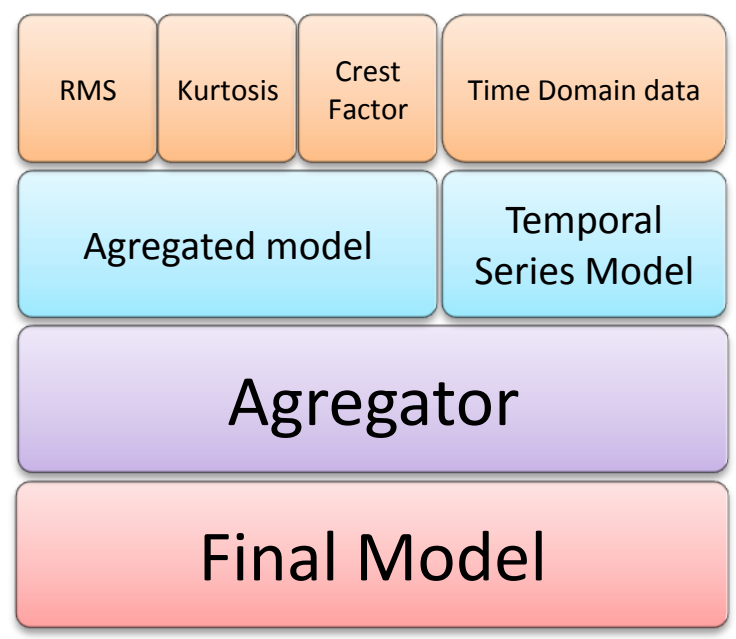

Figure 3 - Classifier cascade by grouping

In the first level, there are the raw data and characteristics extracted from signal time windows. These data and characteristics alone do not predict the condition of a monitored system. Therefore, it is necessary that they are consumed by machine learning models (second level). Models are trained with known non-anomalous observations and then, given a new observation, classify it as normal or anomalous.

The grouper, in turn, receives the classifications of several models on the same observation and determines the final classification. It is important to ensure that the grouping combination process is robust, as inappropriate synthesis can be detrimental to the performance of the model. [15].

The specific group chosen for the development of the work was Locally Selective Combination in Parallel Outlier Ensemble (LSCP) [15]. Being an aggregator classifier, the LSCP initially receives several untrained anomaly detection sub-models. It then conducts individual training for each of them.

The types of sub-models chosen to use LSCP were as follows:

- $\quad \mathrm{kNN}$ (K-Nearest Neighbor) - Anomalous point detection based on the distance from a point to its nearest neighbor at level $\mathrm{k}[16]$.

- LOF (Local Outlier Factor) - Detection of anomalous points based on the principle of local outlier factors, analyzing the density of points, and determining the probability that a given point does not belong to the standard set [17].

- PCA (Principal Component Analysis) - model based on the difference between the values of characteristics of greater weight in the data [18]. 


\section{RESULTS AND DISCUSSION}

Figure 4 shows the results of applying the algorithm to the data set of [12] for channel number 1 of test 2 .

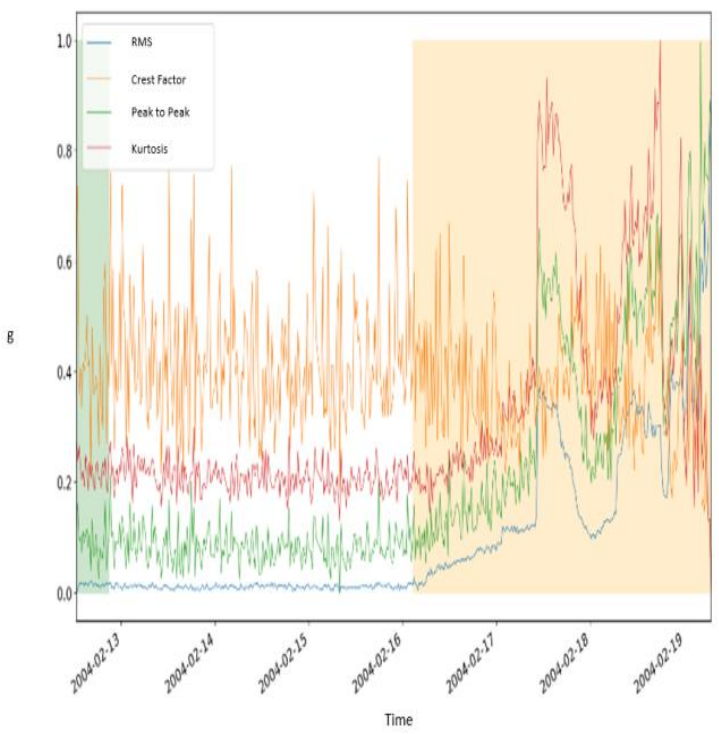

Figure 4 - Raw data, training, and prediction.

The graph shows the calculated values of RMS intensity, crest factor, peak to peak and kurtosis throughout the experiment. The green colored area represents the section used for training the model, and the orange colored area represents the points for which abnormal functioning was detected and an alert was generated. The blank area, therefore, represents the points where the operation was analyzed and considered normal.

For easier visualization of the values of different magnitudes, the values shown were normalized to the range of 0 to 1 , where 1 is the highest intensity reached during the experiment.

For the data window in question, the failure occurred was a defect in the outer ring of the bearing. A gradual increase in RMS, peak to peak and kurtosis values can be observed in the period between 02/16 and 02/17. Approximately in the middle of $02 / 17$, there is a big peak in the measured quantities.

In Figure 4, the model identified the anomaly just before the measured values started to rise gradually, and approximately 36 hours before there was the big peak in machine vibration.

For the data referring to this experiment (channel 1 of test 2 of the dataset), no false positives generated by the model were observed (warning triggers without the operation being in fact anomalous).

The training time used was 51 observations, comprising an interval of 8.3 hours. As the standard for the dataset, observations comprise intervals of 1 second each at $20 \mathrm{kHz}$, spaced 10 minutes apart.

The supervisory system was developed through the integration of the database with Grafana, an open visualization solution. The dashboard with information captured by the system can be seen in Figure 5 .

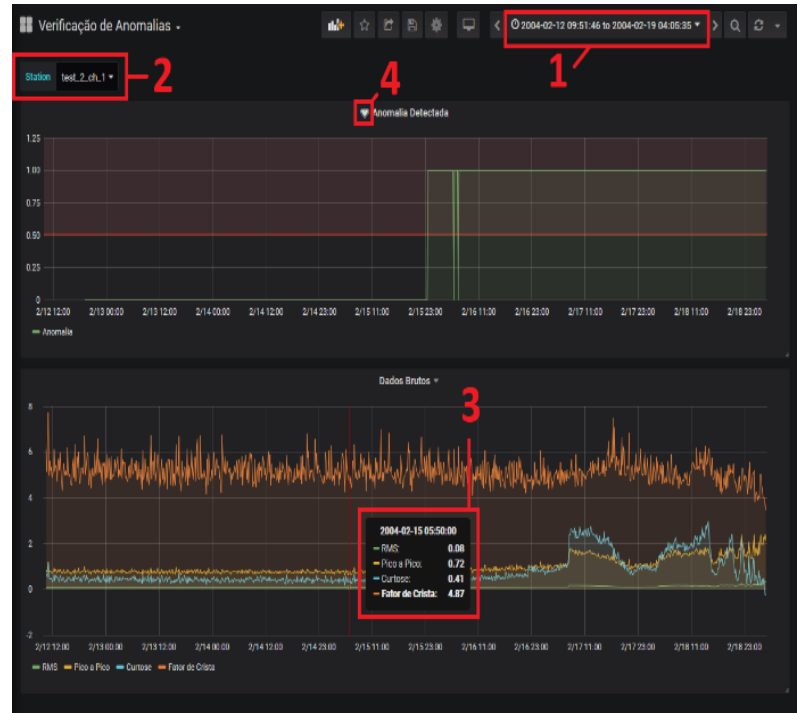

Figure 5 - Vibration data dashboard.

In figure 5 , the raw data and the status, of anomaly detected or not, for the current moment and some previous days can be observed.

The display range can be easily configured to show more details or a longer-term view, using the time selector indicated by marker 1 . There is also the possibility to define a fixed interval, so that, for example, the last two days are always displayed, with automatic update of the visualization.

Marker 2 indicates the monitoring station selector. Each station can represent, for example, a machine, so it is through this control that you define which sensor you want to observe.

Marker 3 shows the detail of raw data that is obtained by placing the mouse over a certain point on the graph. In the small window, the actual data for each quantity variable is shown, and the exact time of that point are is shown.

Marker 4 shows the heart-shaped indicator that indicates that graph is connected to the alert generation system. When passing the red line for a configurable period, an alert is sent by the configured means (for instance, by e-mail).

\section{CONCLUSION}

Throughout the work, the necessary steps to create a fault prediction system with open-source technologies were presented and developed. From the algorithm to the specification of the infrastructure used, open solutions were found that provided a result that in the eyes of the author was satisfactory.

The results obtained by the algorithm were good when it came to predicting failures in stages that are not obvious to a human being, and the platform was developed in such a way that the system could be easily adapted to a large number of sensors / machines. Due to the open nature of the libraries and tools used, there is no monetary impediment to anyone who is inspired to create a similar system.

Future works that come to address a similar theme may develop, as a suggestion, customizations of the algorithms used at a lower level; perhaps with libraries such as TensorFlow or Pytorch, which present machine learning tools through neural networks, different from the statistical methods used by the present work. At the cost of the simplicity of the model, greater nuances can be captured in the anomaly detection process. 


\section{REFERENCES}

[1] Marcorin, W. R., \& Lima, C. R. (2003). Análise dos Custos de Manutenção e de Não-manutenção de Equipamentos Produtivos. Revista de Ciência \& Tecnologica, 11(22), 35-42.

[2] Lebold, M., McClintic, K., Campbell, R., Byington, C., \& Maynard, K. (2000). Review of Vibration Analysis Methods for Gearbox Diagnostics and Prognostics. 54th Meeting of the Society for Machinery Failure Prevention Technology, (pp. 623-634). Virginia Beach.

[3] Otani, M., \& Machado, W. V. (2008). A proposta de desenvolmento de gestão da manutençãoindustrial na busca da excelência ou classe mundial. Revista Gestão Industrial, 4(2), 1-16.

[4] Pandiyan, J., Umapathy, M., Balachandar, S., Arumugam, M., Ramasamy, S., \& Gajjar, N. C. (2006). Design of Industrial Vibration Transmitter Using MEMS Accelerometer. International MEMS Conference (pp. 442-447). Gujarat: Institute of Physics Publishing.

[5] Heng, A., Zhang, S., Tan, A. C., \& Mathew, J. (2009). Rotating machinery prognostics: State of the art, challenges and opportunities. Mechanical Systems and Signal Processing(23), 724-739.

[6] Paudyal, S., Atique, M. S., \& Yang, C. X. (2019). Local Maximum Acceleration Based Rotating Machinery Fault Classification Using KNN. IEEE EIT 2019. Grand Forks.

[7] Cubillo, A., Perinpanayagam, S., \& Esperon-Miguez, M. (2016). A review of physics-based models in prognostics: Application to gears and bearings of rotating machinery. Advances in Mechanical Engineering, 8(8), $1-21$.

[8] Stringer, D. B., Sheth, P. N., \& Allaire, P. E. (2012). Physics-based modeling strategies for diagnostic and prognostic application in aerospace systems. Journal of Intelligent Manufacturing(23), 155-162.

[9] Jia, X., Huang, B., Feng, J., Cai, H., \& Lee, J. (2018). Review of PHM Data Competitions from 2008 to 2017: Methodologies and Analytics. Annual Conference of the Prognostics and Health Management Society, (pp. 1-10). Cincinnati.
[10] Ahmad, R., \& Kamaruddin, S. (2012). An overview of time-based and condition-based maintenancein industrial application. Computers \& Industrial Engineering(63), 135-149.

[11] Labib, A. W. (2004). A decision analysis model for maintenance policy selection using a CMMS. Journal of Quality in Maintenance Engineering, 10(3), 191-202.

[12] Qiu, H., Lee, J., Lin, J., \& Yu, G. (2006). Wavelet filterbased weak signature detection method and its application on rolling element bearing prognostics. Journal of Sound and Vibration(289), 1066-1090. Acesso em 2019, disponível em https://ti.arc.nasa.gov/tech/dash/groups/pcoe/prognosticdata-repository/

[13] Gupta, M., Gao, J., Aggarwal, C. C., \& Han, J. (2014). Outlier Detection for Temporal Data: A Survey. IEEE Transactions on knowledge and data engineering, 26(9), 2250-2267.

[14] Krawczyk, B., Minku, L. L., Gama, J., Stefanowski, J., \& Wozniak, M. (2017). Ensemble learning for data stream analysis: A survey. Information Fusion(37), 132-156.

[15] Zhao, Y., Nasrullah, Z., Hryniewicki, M. K., \& Li, Z. (2019). LSCP: Locally Selective Combination in Parallel Outlier Ensembles. SIAM International Conference on Data Mining. Calgary: Society for Industrial and Applied Mathematics.

[16] Ramaswamy, S., Rastogi, R., \& Shim, K. (2000). Efficient algorithms for mining outliers from large data sets. ACM SIGMOD international conference on Management of data (pp. 427-438). Dallas: ACM New York.

[17] Breunig, M. M., Kriegel, H.-P., Ng, R. T., \& Sander, J. (2000). LOF: Identifying Density-Based Local Outliers. $A C M$ SIGMOD International Conference on Management of Data. 2, pp. 93-104. Dallas: ACM New York.

[18] Shyu, M.-L., Chen, S.-C., Sarinnapakorn, K., \& Chang, L. (2003). A Novel Anomaly Detection Scheme Based on Principal Component Classifier. IEEE Foundations and New Directions of Data Mining Workshop, (pp. 172179). 